\title{
Art, science, and the existential focus of clinical medicine
}

A Warsop

The continuing debate over the status of medicine as an art or a science remains far from resolved. The aim of this paper is to clarify what is meant by the art of medicine. In the following interpretation I contrast two current perspectives of the medical art. I argue that the art of medicine is best understood in terms of the Aristotelian notion of techne. It consists of listening skills directed to the lived experience of the patient in such a way that knowledge (principally scientific knowledge) can be applied in a therapeutic way. This constitutes what I call medicine's existential focus. The art of medicine is prior to and independent of medical science which plays an important but subordinate role.

\section{M} any writers have argued that art and literature should have a place in the medical curriculum on the grounds that art helps doctors to understand experiences of illness and that art itself can fulfil a therapeutic role. ${ }^{1}$ But is there anything intrinsic to medical practice that can reasonably be called an art? Is reference to "art" merely grandiose obscurantism (as in the "secret art" of the healer) or is the reference to art meant in the aesthetic sense? Or, most likely, is art used in the sense of learned skill or craft? These sorts of questions cannot be answered by empirical research but require philosophical analysis. My method will be an interpretation of the phenomenon of clinical practice. This involves describing aspects of that practice and making explicit the assumptions which are found to underlie it and render it intelligible. As my point of departure I discuss two contrasting views of the art of medicine. I will go on to discuss the nature and role of scientific reasoning in medicine, with reference to a case study. I will then go on to describe how the ancient Greek notion of techne is presupposed in clinical practice. The notion of techne gives sense to the art of medicine and, in combination with what I call the "existential focus" of medicine, militates against it being a science or even an applied science in any straightforward way.

\section{TWO CONTRASTING VIEWS OF THE ART OF MEDICINE}

Whatever the art of medicine is, most people would agree that it refers, first and foremost, to a kind of activity carried out by medical practitioners. In his informative article, Saunders ${ }^{2}$ emphasises this point, but he goes on to say that medicine's art is not exclusively described by clinical performance. According to him, medicine is primarily applied science which requires what he calls a "doctrine of standard empiricism". This is described as a mode of inquiry the aim of which is to produce "objective knowledge and truth and to provide explanations and understanding". On this account, it is the community of science which establishes the required impersonal objective character by means of intersubjective agreement concerning reliable scientific demonstration. But, as Saunders points out, this is not the whole story, the practice of medicine requires making value judgments in particular situations and these judgments cannot be fully explicated under the rubric of science. There is a "common culture" within clinical medicine in which doctors participate. By a process of learning within this common culture doctors develop a capacity to reflect on diverse scientific evidence and to apply it in a particular clinical context. He concludes that the art of medicine is integral to the culture of medicine as applied science.

Saunders's view, as I take it, is that there are various kinds of activities undertaken by doctors which, though not scientific, are essential to the practice of medicine as a science. These sorts of activities are contrasted with evidence based medicine and are, on his view, crucial to its implementation. They collectively constitute the art of medicine. He illustrates what he means with two sorts of examples, first the use of "rules of thumb" in medical practice, and second, the kinds of reflective activities required when dealing with uncertainty in a clinical context. He brings the salient issues to light by giving an account of a case study reported elsewhere. ${ }^{3}$ A 42 year old mother of two small girls, despondent over job difficulties, is contemplating genetic screening for breast cancer as she approaches the age at which her mother was diagnosed with the disease. The issues to be addressed by the doctor include the following: concerns to do with the relevance of scientific data to this situation; questions to do with doctor patient relationship issues, and, most importantly, questions to do with one's knowledge of the patient and oneself. The questions that a good doctor would formulate in order to address this situation might include the following:

- How much should I trust the available information?

- Would knowing the results make a difference?

- What approach would be most helpful to the patient?

- To what extent is the patient's desire for testing biased by her fears, depression, or incomplete understanding of the illness and test?

- What values and biases affect the way I frame this situation for myself and for the patient? 
These are the issues that come into play whenever a clinical judgment is made, and they constitute the context of any clinical decision.

In his article Saunders compares his own account favourably with that given by Lloyd H Smith Jr in Cecil Textbook of Medicine. The latter characterises the art of medicine in the following way:

\section{In the art of medicine the physician must be the advocate of the patient as well as the adversary of disease. ${ }^{4}$}

For Smith, the art of medicine is conceived as the activity of patient advocacy by means of what is called "the instrument of human faculty". This is a term coined by a famous neurosurgeon, Trotter, to encompass a range of appropriate activities such as the ability to listen, to inform, and to maintain solidarity with the patient throughout adversity. The most important of these is the ability to listen in such a way that one discerns and responds to the needs of the patient. As Trotter says: "disease often tells its secrets in casual parenthesis". The ability to listen to patients is obviously central to the clinician's work and the contention is that it is a skill or art that can be learned.

With his emphasis on the physician being the advocate of the patient, Smith implies that the primary feature of the art of medicine is patient care. Although Smith does not explicitly say so, the logic of the activity of care implies two crucial features; first, care has an object. Care is necessarily care of something. In the clinical situation it is care of some particular patient in some particular context. Second, care is a goal directed activity-it is concerned with bringing about a certain (favourably perceived) situation or state of affairs. In medicine, this is the wellbeing of the patient.

How do the two accounts compare? Smith's approach is humanitarian, with the emphasis on care in a clinical context. Notably, the notion of "human faculty" is developed independently of any reference to science. He gives priority to the actual human relationships in a clinical encounter. Saunders, although he brings out the experience of clinical encounters with the case study, emphasises that the art of medicine is "not just practical performance". He sees the art of medicine as part of a much wider notion; it is part of the culture of medical science and includes all those unquantifiable aspects of clinical reasoning which are essential to its application as science. On his account the art of medicine is not something confined to the actual clinical encounter but includes a range of activities which fill the gap between the ideal, theoretical construct of randomised controlled trials and the actual clinical world. Saunders sees the art of medicine as part of the culture of science, whereas Smith sees the role of science as subordinate to the humane art of listening and advocacy.

\section{SCIENTIFIC REASONING IN THE MEDICAL CONTEXT}

To understand the relative role of art and science in medical practice it would be helpful to clarify the role of scientific reasoning in clinical work. For the clinical process to be one of pure scientific reasoning, we can say that certain criteria must obtain. First, a hypothesis must be formulated which is proposed to explain a set of data. A hypothesis in science is a general statement or theory derived from particular data and from which particular inferences can be deduced. So, from the proposed theory "all planets have elliptical orbits", given the information that Mars is a planet, we can deduce that Mars has an elliptical orbit. Second, once the hypothesis has been formulated, experiments are conducted to test it. In this case, observations of Mars's orbit serve to confirm or falsify the hypothesis under question. This is the process of scientific reasoning as described by Popper's hypotheticodeductive method. ${ }^{5}$ So what happens in actual clinical practice?
It might be reasonable to suggest that the clinician as medical scientist proceeds by formulating a number of possible explanatory hypotheses which are proposed as solutions to a clinical problem. These hypotheses are then tested in the light of empirical evidence. The culmination of this scientific process of diagnosis is an act of judgment in which the (hopefully) correct hypothesis is selected. Going back to the case of the woman considering breast screening, what is the hypothesis here? The clinical problem to be answered is: is it the case that the woman should undergo genetic screening? The difficulty here is that the clinical problem does not seem to be readily amenable to solution by the scientific method as I have described it. First, it is clear that in general, the process of diagnosis does not closely resemble that of constructing scientific hypotheses. What happens in the clinical process is that patients are judged to be instances of certain disease concepts. The clinician identifies the case as a case of, say, genotype $\mathrm{BC} \mathrm{Al}$. He does this by listening closely to the patient and then conducting an appropriate examination and investigation based on the patient's history. This is scientific thought but science subordinated to the art of listening. In marked contrast to the pure scientist he does not just observe clinical cases and then construct a disease concept to explain them. Such an approach would be paradigmatically unethical. Second, the activity of diagnosis is only a very limited part of the doctor's task. There is no "correct" answer to the question of whether the 42 year old should be screened or not, everything depends on the patient's own (subjective) wishes and the clinical context. The doctor's role here is to marshall the relevant facts to empower the patient so that she can make an informed decision. Even if it is the case that the diagnostic process involves scientific reasoning, we still need to distinguish the question of diagnosis from the question that is always present in the cliical encounter and not so easily formulated as a testable hypothesis. This is: "What is to be done for this person?" In clinical work it is the latter question which takes priority over the progress of scientific knowledge.

\section{MEDICINE AS TECHNE}

The ancient Greek concept of techne is fundamental to understanding what art is. Perhaps if clinical practice can be shown to exhibit techne then there is a basis for understanding it as an art. Techne describes a fundamental and defining feature of humans - the capacity of thought to inform productive action. Its discovery is, according to Gadamer, ${ }^{6}$ nothing less than the event that marked the first step to what now characterises Western civilisation. Techne is a special form of practical knowing which is concerned with making things. It is the capacity to undertake tried and reasoned activity culminating in the production of a work or an ergon. In order for some action to exemplify techne it does not have to be done with the explicit goal of providing an explanation for something or contributing to a body of knowledge. What is essential is that 1) the origin of the ergon is a human maker whose techne releases a new entity; and, 2) the ergon figures in the explanatory ground for the techne and constitutes its goal. Thus, arts and crafts/skills are prime examples. The goal of the cabinetmaker is to produce a cabinet and the goal of the sculptor is to produce a sculpture-a work of art. The cabinet and the sculpture, respectively, serve to confirm and explain these activities. These sorts of activities (involving production) only become intelligible in the light of their goals.

How can this notion illuminate the art of medicine? The kinds of activities described by Smith and Saunders are, by common agreement, essential to medicine but they are not, according to the criteria of the "hypotheticodeductive" method described above, scientific. That is not to say that they are not technical, however. They seem to be a sort of techne in that they require learned clinical skills that may be compared to those of craftsmen. As Gadamer notes, the newly restored 
health of the patient can be viewed as the ergon of this activity. ${ }^{7}$ It is this production of the actual healthy patient that explains, justifies, and renders intelligible all the antecedent clinical activities just as the production of a functioning table renders intelligible the activities of the carpenter. Without the goal of newly restoring the patient (in some sense) the doctor's activities are unintelligible. The activity of the doctor and the craftsman is goal directed and characterised by care for the ergon they aim to produce using their respective skills. Thus, when understood as a form of techne, the notion of care as attentive advocacy implicit in Smith's/Trotter's account becomes not merely care as emotional concern but care in a truly technical, skillful sense. Aristotle contrasts techne with phronesis or practical wisdom in how one conducts oneself. ${ }^{8}$ Techne aims at an end other than itself and so production has its value in what is produced, whereas action governed by practical wisdom has its end in itself because the end is merely doing well. The value of the carpenter's skill, like that of the doctor's, is judged not by the activity itself but by the ergon it produces. The new table and the restored patient, respectively, are testimony to their skill and care.

Clinical work understood as techne also helps to characterise the day to day work of the doctor. Medicine is characterised by the doctor/patient consultation. Techne finds expression in episodes of action which end when an ergon is complete. The way in which the activity of medicine consists of a series of clinical episodes contrasts sharply with the activity of the scientist. Pure science never "ends" in the way a clinical episode does. It proceeds by postulating hypotheses or general theories. Such hypotheses do not apply just to the data currently being dealt with in a particular experiment but they are constructed to apply generally to all items of a certain kind. The hypothesis that all planets have elliptical orbits-for example, serves to explain the movements of all planets-those observed and those as yet unobserved. What then happens is that experiments/observations (of other planets) are conducted with the express purpose of testing the hypothesis. The hypothesis is corroborated in so far as it survives this testing but it is never proven. The work of the scientist, unlike the work of the doctor, is never complete. The problem is that the hypothesis is never proven because "new" data may emerge which will falsify it.

Medicine, in contrast, has a more concrete human end point. Diagnostic hypotheses are constructed in response to the findings of skilful history taking and examination. They apply to a particular patient in a particular contextdiagnoses belong to single individuals and they are not supposed to be general explanatory theories. They serve to organise and explain a single patient's salient problems in such a way that these problems can be improved. They are taken to be confirmed by empirical testing-the biopsy of a breast lump confirms the diagnosis of carcinoma and at that point the investigation is taken to be complete. In contrast to "pure" scientific investigation further diagnostic tests are not undertaken to "test" the initial hypothesis. The diagnosis directs the physician to the appropriate treatment and the initial hypothesis is confirmed (or not) by the patient's clinical progress. When the different characters of the work of scientist and clinician are demonstrated in this way, it is clear that the clinical activities of medicine seem to resemble a kind of techne conceived as skill or craft, but they fail to resemble the work of pure science.

\section{MEDICINE'S EXISTENTIAL FOCUS}

There are some qualifications to understanding medicine in terms of techne. First, although fine art is an example of techne, it seems clear that the art of medicine cannot be art in any aesthetic sense. The "cured" healthy patient cannot reasonably be considered as a created work of art. The goal of the artist is to create a work of art specifically for the purpose of providing aesthetic pleasure. It would obviously be perverse and unethical for a doctor to treat his patient in the same way. The goal of medicine is to produce wellbeing or health for the sake of the patient and not for the sake of art. We must distinguish between aesthetic goals instrumental to the primary goal of restoring health and aesthetic goals as such. The cosmetic surgeon takes aesthetic factors into consideration as part of her daily work, but such factors are subsumed by the priority to restore her patient's health. If the cosmetic surgeon were to tell us that her goal was to create art, using her patient as raw materials as a potter uses clay, we would be decidedly uneasy about her fitness to practise. The production of health or wellbeing is not instrumental to some other goal, aesthetic or otherwise, but is to be pursued for the patient's sake. We must conclude, then, that medicine fails to qualify as an art in the sense of art understood as fine art, as say painting or sculpture-for example.

Second, in contrast to other examples of techne, medicine is patently different in so far as the ergon is a person. Let's go back to Saunders's example and look at the phenomenon of clinical work as it presents itself to us. He describes a situation where a 42 year old mother of two consults her physician, contemplating genetic screening for breast cancer. He discerns that there a number of essential non-scientific questions which arise in this situation (see above). What makes the medical encounter very different from other sorts of techne is that the doctor's concerns are existential and not merely technical. By this I mean that it is the human concerns of the patient-her problems as they are lived and experiencedwhich initiate and determine the progress of the clinical encounter. The doctor/patient encounter-the consultation"sets the agenda" for the doctor's techne in a way in which the carpenter's goal of the finished table does not constrain the actions of the carpenter. While both doctors and carpenters care for their work in the way characteristic of techne, the patient's lived problems convey a special urgency and character to the doctor's work. We could express this by saying that the object of the doctor's care (the lived experience of the patient) is ontologically distinct from the object of the carpenter's care (the inanimate wood with which he works). The theme underlying the questions raised in Saunders's example is to do with how the doctor relates his scientific knowledge to the clinical problem as it is lived by the patient. The distinguishing feature of the doctor's techne is the capacity to attend to the patient's lived experience in such a way that scientific knowledge can be brought to bear to restore the patient's wellbeing. The goal directed care of the doctor's techne is patient care, understood in this existential sense. This is medicine's existential focus.

One objection to the argument put forward here might be to say that even if medicine fails to qualify as a pure science it must be an example of an applied science. In one sense this is patently correct. Medical treatments and diagnostic methods often employ highly sophisticated science. But this is not the same as saying medicine is just applied science, nor does it mean that the medical art is subordinate to science. This is apparent when we consider medicine's existential focus and the way in which the doctor's concerns are ontologically distinct from those of the applied scientist. One might want to suggest that an orthopaedic surgeon, when replacing a knee joint, say, is just doing applied physics, just as an engineer is doing applied physics. Of course, the biomechanical engineer who designs and builds the artificial knee is doing applied physics but is that really true of the surgeon? The analogy fails when we reflect on the fact that the surgeon's work, in common with that of every other doctor, is determined by the lived experience of the patient. Suppose Mrs Smith has advanced osteoarthritis affecting her left knee. Further suppose that Mrs Smith's orthopaedic surgeon replaces her left knee with an artificial one. If Mrs Smith finds that six months after the operation she is still unable to manage her 
stairs at home because of persistent left knee pain, then medical review is required. The outcome of the clinical episode does not depend merely on the physical properties of the artificial knee but on the artificial knee as it is experienced by the patient. The biomechanical engineer does not have to concern himself with Mrs Smith's pain when stair climbing whereas the surgeon does. He must relate his specialist knowledge to the patient's lived experience in order to determine the source of the problem and what needs to be done. The existential focus of medicine means that the doctor's job is always more than restoring function in just biochemical, anatomical, or physiological terms. It requires that the patient is seen and evaluated as an experiencing person and not just as an aggregate of scientific processes (as would be the case if doctors did just applied science and no more).

Science is, of course, essential to medicine but medicine cannot be simply identified with pure science nor even with applied science. I have argued that the art of medicine is essentially composed of the clinical skills of listening and advocacy brought to bear in the consultation. These skills can be understood as a special kind of techne, distinguished by an existential focus directed to and caring for the patient's lived experience in such a way that science can be applied therapeutically. In this sense, Trotter's notion of the "instrument of human faculty" is as central to the work of the clinician as it ever was. Rather than medicine's art being part of a culture of medical science, science can only play its part if doctors are effective practitioners of the art of medicine.

\section{FUNDING}

The writing of this paper was partly supported by the East London and Essex Network of Researchers.

\section{REFERENCES}

1 Smith B, Taylor R. Medicine-a healing or a dying art? British Journal of General Practice 1996;46:249-51

2 Saunders J. The practice of clinical medicine as an art and as a science. Journal of Medical Ethics: Medical Humanities 2000;26:18-22.

3 See reference 2: 21

4 Smith LH. Part 1. Medicine as an art. In: Wyngaarden JB, Smith LH, eds. Cecil textbook of medicine [18th ed]. Philadelphia: WB Saunders, 1985: 2.

5 Popper KP. The logic of scientific discovery. London: Unwin/Hyman, 1959.

6 Gadamer H-G. The enigma of health. Cambridge: Polity Press, 1996: 31-44.

7 See reference 6: 32 .

8 Aristotle. The Nicomachean ethics. Harmondsworth: Penguin, 1976: 208-9.

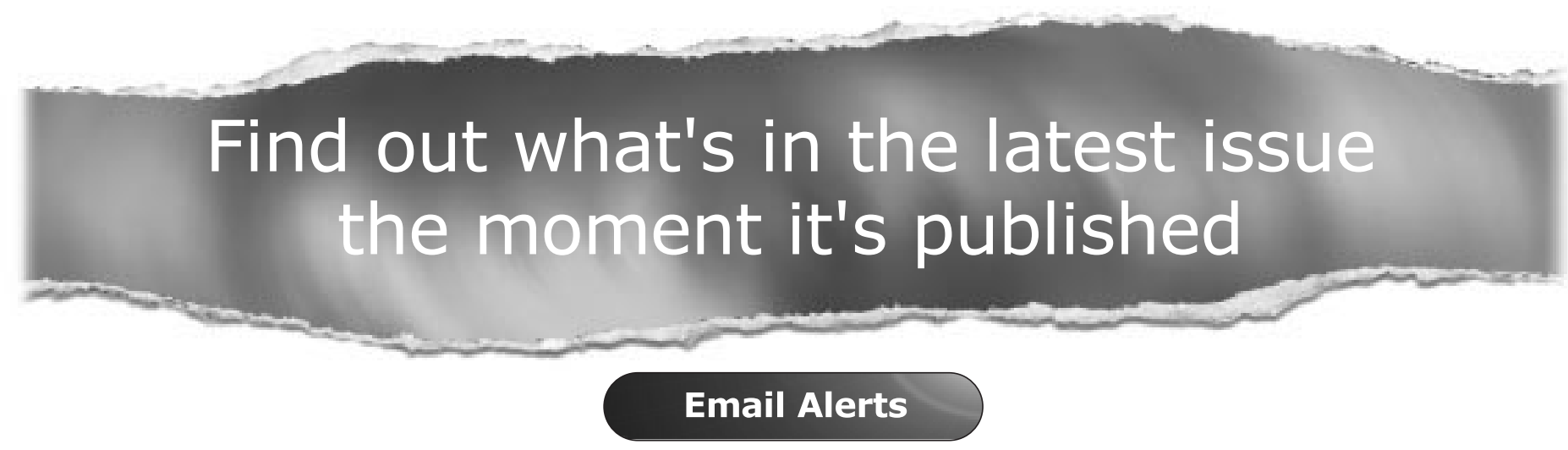

Sign up to receive the table of contents by email every month. You can select from three alerts: Table of Contents (full), TOC Awareness (notice only); Medical Humanities related announcements.

\section{www.medicalhumanities.com}

